traZeneca, Consultant for: Pfizer, AstraZeneca, Proteothera, Celgene, Horizon, P. Khanna Grant/research support from: AstraZeneca, Consultant for: Horizon, Ironwood, Selecta Bio, A. Yeo Consultant for: Horizon Pharma, P. Lipsky Consultant for: AstraZeneca, Celgene, EMD Serono, GSK, Horizon Pharma, Janssen, Medimmune, Pfizer, Roche, Sanofi, UCB

DOI: 10.1136/annrheumdis-2017-eular.4114

\section{THU0450 HOSPITAL READMISSIONS FOR GOUT IN THE UNITED STATES: A NATIONAL DATABASE STUDY}

N. Annapureddy ${ }^{1}$, A. Saha ${ }^{2}$, P. Poojary ${ }^{3,4}$, K. Chauhan ${ }^{4}$, G. Nadkarni ${ }^{4}$ ${ }^{1}$ Division of Rheumatology and Immunology, Vanderbilt University Medical Center, Nashville; ${ }^{2}$ Department of Public Health; ${ }^{3}$ Department of Public Health;

${ }^{4}$ Division of Nephrology, Icahn School of Medicine at Mount Sinai, New York, United States

Background: Gout is associated with significant burden and risk of readmission. Little is known about readmissions among Gout patients on a national level in the United States.

Objectives: The aim of this study was to describe unplanned hospital readmission rates among adult gout patients and assess predictors of readmission.

Methods: We analyzed the 2013 National Readmission Database (NRD) to quantify readmission rates among Gout patients. The NRD includes weighted discharge data from 21 geographically diverse states accounting for $49.3 \%$ of the U.S. population. It includes approximately 14 million un-weighted discharges (49.1\% of all U.S. discharges) corresponding to 36 million annual discharges nationwide. NRD data is from patients with non-Medicare payers (Medicaid, private, self-pay, or other). Gout hospitalizations were identified using the International Classification of Diseases, ninth Revisions, Clinical Modification (ICD-9-CM) diagnosis code 274.0x. All hospitalizations for patients age $\geq 18$ were included. In efforts to exclude routine readmissions, we excluded those admissions related to pregnancy, those for chemotherapy, admissions where the patient was readmitted the same day as they were discharged, who had deaths during the same index hospitalizations, hospitalizations for less than 24 hours, and those with missing discharge. We utilized Chi-square tests, $t$ tests and Wilcoxon rank-sum tests as appropriate. Survey logistic regression was used to assess the relationship between potential predictors for readmissions and the odds of at least one 30-day unplanned readmission. This analysis was chosen given the NRD data, which involves nested, weighted observations that are inherently stratified in clusters to produce national estimates.

Results: A total of 10708 index hospitalizations which had Gout as the primary diagnosis were included in the analysis. Among those with a primary Gout diagnosis, there were 1212 30-days readmissions (11.3\%). 14.3\% percent of patients with Gout as the primary diagnosis on index hospitalization were readmitted with the same diagnosis. The next most common readmission diagnoses were congestive heart failure $(\mathrm{CHF})$, septicemia, and acute and unspecified renal failure (Figure $1 \mathrm{~A}$ ). In multivariable analysis of index hospitalizations with Gout as the primary diagnosis, $\mathrm{CHF}$ (OR $1.25,95 \% \mathrm{Cl}, 1.05-166)$, chronic kidney

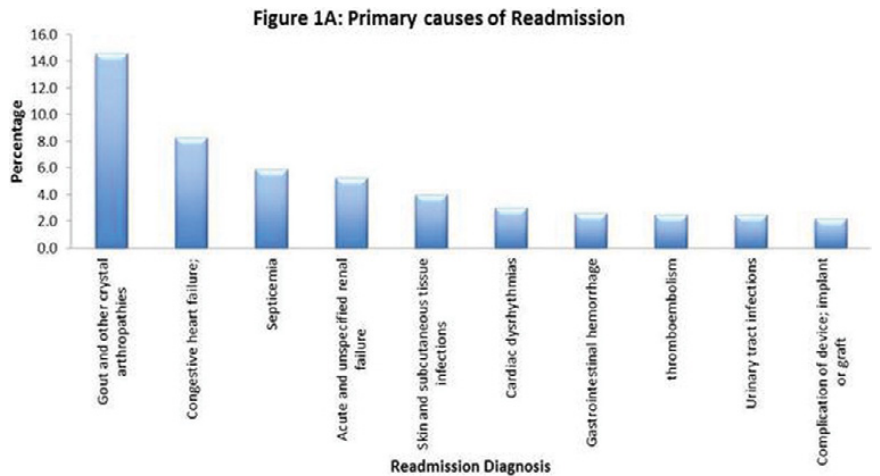

Figure 1B: Predictors for Primary Gout Readmissions

\begin{tabular}{|c|c|c|c|c|}
\hline Patients Characteristics & & OR & $(95 \% \mathrm{Cl})$ & P-value \\
\hline Congestive Heart Failure & $1-1$ & 1.25 & $(1.05-1.66)$ & 0.04 \\
\hline Chronic Kidney Disease & $\longmapsto$ & 1.54 & $(1.23-1.92)$ & 0.0002 \\
\hline Atrial Fibrillation & $1-1$ & 1.33 & $(1.10-1.78)$ & 0.05 \\
\hline Deep Vein Thrombosis & $=-1$ & 1.29 & $(1.03-1.88)$ & 0.04 \\
\hline APR-DRG Severity Level 3 & $=\quad-1$ & 1.51 & $(1.01-2.38)$ & 0.04 \\
\hline APR-DRG Severity Level 4 & . & 2.10 & (1.06-4.58) & 0.05 \\
\hline Discharge to Specialized Care & $\longmapsto$ & 1.47 & $(1.07-2.02)$ & 0.02 \\
\hline Discharge to Home Health Care & $-\infty$ & 1.35 & $(1.03-1.77)$ & 0.03 \\
\hline Discharge against Medical Advice & $\longmapsto$ & 3.85 & $(1.50-9.91)$ & 0.005 \\
\hline & 1 Adjusted Odds Ratio & & & \\
\hline
\end{tabular}

disease (CKD) (OR 1.54, 95\% Cl, 1.23-1.92), atrial fibrillation (AF) (OR 1.33, 95\% $\mathrm{Cl}, 1.10-1.78$ ), deep venous thrombosis (DVT) (OR 1.29, 95\% Cl, 1.03-1.88), APR-DRG severity level 3 and 4 (OR 1.51, 95\% Cl, 1.01-2.38 and OR 2.10, $95 \% \mathrm{Cl}, 1.06-4.58$ ), discharge to specialized care (OR 1.47, 95\% Cl, 1.07-2.02), discharge to home health care (OR 1.35, 95\% Cl, 1.03-1.77), and discharge against medical advice (OR $3.85,95 \% \mathrm{Cl}, 1.50-9.91$ ), were significantly associated with 30-days readmission after adjusting for demographics, comorbidities, hospital characteristics, payer type, and the APR-DRG severity scale (Figure 1B). Conclusions: In a national readmissions database, $11.3 \%$ of patients admitted with a primary diagnosis of Gout were readmitted within 30 days. Significant predictors of readmission included CHF, CKD, AF, APR-DRG severity level 3 or 4 and any discharge other than routine.

Disclosure of Interest: None declared

DOI: 10.1136/annrheumdis-2017-eular.6468

\section{THU0451 A METHOD FOR COUNTING CALCIUM PYROPHOSPHATE CRYSTALS IN THE SYNOVIAL FLUID}

P. Montagna, R. Brizzolara, S. Soldano, M. Cutolo, M.A. Cimmino. Department of Internal Medicine, University of Genova, Genova, Italy

Background: Identification of calcium pyrophosphate dihydrate (CPP) crystals in the synovial fluid (SF) from inflamed joints provides a definitive diagnosis of CPP deposition disease (CPPD) (1). CPP crystals may also be found in non-inflamed joints, allowing diagnosis also during asymptomatic periods (2). SF analysis and CPP crystals count could be used to evaluate disease activity during follow-up. It is more difficult than the count of monosodium urate (MSU) crystals, already tested in a previous work (3), for CPP crystals show different shapes, are often very minute and non-birefringent.

Objectives: To study an objective method for counting CPP crystals in the SF. Methods: The SFs aspirated from the knees of 15 consecutive patients ( 8 men) affected by CPPD diagnosed according to the EULAR definition were analysed. Cytological evaluation included SF leukocyte and differential count. For crystal detection, a small drop of fresh SF was placed on a glass slide and examined by compensated polarized microscopy $(400 \mathrm{x})$. To facilitate crystal count, the slide was divided into 4 equal parts drawing a cross with a pencil. The count was performed by continuous viewing and for each field both the number of birefringent and non-birefringent crystals was noted. Two observers evaluated separately 6 SFs and repeated the count after 24 hours. SFs were divided into 4 groups: SFs with $<50$, from 50 to 400 , from 401 to 1200 , and $>1200$ crystals.

Results: Mean time needed for the count was 60 minutes. Inter-reader agreement was $0.68(0.47-0.88)$ for CPP crystals, $0.68(0.50-0.85)$ for the birefringent ones and $0.60(0.38-0.81)$ for the non-birefringent. Intra-reader agreement was 0.48 $(0.17-0.78)$ for the first examiner and $0.30(0.14-0.74)$ for the second. In 7 patient the SF was aspirated from an inflamed knee. Crystal number did not correlate with the presence of knee inflammation $(r=0.41 ; p=0.19)$, the SF volume $(r=0.14 ; p=0.61)$, the number of leukocytes $(r=0.36 ; p=0.19)$, the $\%$ of PMN $(r=0.25 ; p=0.37)$, and the presence of intracellular crystals $(r=0.31, p=0.27)$. Actively inflamed joints had a higher SF volume [11 ml $(10-20 \mathrm{ml}) \mathrm{vs} .2 \mathrm{ml}(1-10$ $\mathrm{ml}), \mathrm{p}=0.03]$ and a higher percentage of PMN $[72 \%(0-94 \%)$ vs. $12 \%(0-68 \%)$, $\mathrm{p}=0.028]$. SF with intracellular crystals showed also a higher percentage of PMN (57.1\% $\pm 34.3 \%$ vs. $3 \% \pm 6 \% p=0.006)$.

Conclusions: Our preliminary results indicate that CPP crystal count is less reliable and more time-consuming that that of MSU crystals. Non-birefringent crystals show lower inter-reader agreement than birefringent ones.

References:

[1] Pascual E. J Rheumatol 1996; 35:306-8

[2] Martinez A. Arthritis Rheum 1995; 38:S 246.

[3] Montagna P. Reumatismo 2015; 67(1):29-32.

Disclosure of Interest: None declared

DOI: 10.1136/annrheumdis-2017-eular.5045

\section{THU0452 IMMEDIATE AND LONG TERM EFFICACY OF ANAKINRA IN ACUTE FLARES DUE TO HYDROXYAPATITE CALCIFICATIONS: A REAL-LIFE EXPERIENCE OF 13 CASES}

P. Zufferey, R. Valcov, A. So. DAL, CHUV, Lausanne, Switzerland

Background: Calcifications composed of hydroxyapatite (HA) crystals can induce acute and severe pain accompanied by signs of acute inflammation. In a previous pilot study, we have shown that anakinra was effective in acute flare of calcific periarthritis of the shoulder in the short term

Objectives: The goal of this retrospective observational study was to confirm these results in a larger set of patients, extending the observation to other localizations and to report on the long-term follow-up.

Methods: All consecutive patients with an acute flare due to HA deposition and treated with anakinra between March 2011 and November 2016 were included. Flare was defined as symptoms of acute pain at rest present for $<10$ days. None of the patients had corticosteroid therapy in the last 2 weeks, none had responded to at least 48 hours of high doses of NSAIDs or other rheumatologic diseases explaining the symptoms. Clinical evaluation consisted of patient assessment of pain by VAS (10mm scale) at days $0,1,3,21$ and joint mobility. CRP and ESR measurements, ultrasound and $\mathrm{x}$-ray examinations were performed before the 